experiments Weiss has just reported in Virology is the way in which he blocked DNA synthesis and mitosis of the infected cells. Instead of exposing chick cells to an inhibitor he plated freshly trypsinized chick cells, infected with Rous sarcoma virus, onto confluent monolayers of either mouse cells or chick cells. When mouse cell monolayers are used the chick cells do not replicate their DNA or divide, neither do they develop into foci of transformants. By contrast the infected chick cells plated on top of chick cell monolayers do divide and do develop into foci without the virus replicating in the monolayer cells.

Weiss believes that these findings are consonant with the quantal mitosis idea and he has an equally fashionable explanation for the differential inhibition of chick cell division by monolayers of chick and mouse cells. He suggests, à la Burger, that the mouse cells elaborate and secrete some cell surface component which is stripped from chick cells when they are exposed to trypsin and which is required to keep the cells susceptible to those growth regulatory signals which act in dense cultures to inhibit cell division. Freshly trypsinized chick cells plated onto a monolayer of mouse cells pick up this surface component and their multiplication becomes inhibited. By contrast, Weiss envisages that monolayers of chick cells do not secrete this component.

Be that as it may, Weiss is now hunting mutant Rous viruses which are conditionally sensitive to the density dependent growth of their hosts. Kawai and Hanafusa (ibid., 470) who have also joined in the search for temperature sensitive mutants of Rous sarcoma virus have come up with one mutant Ts 68 which is more or less identical to the mutant isolated and described by Martin (Nature, 227, 1021; 1970). Both the Martin and the Kuwai and Hanafusa mutants appear to define a Rous sarcoma virus gene which specifies a protein that is not a component of the virion but is required to maintain the transformed cell phenotype.

Progress towards the identification and isolation of this crucial viral protein will inevitably be tantalizingly slow, but analysis of the Rous virion proteins is progressing. Rifkin and Compans (ibid., 485), for example, have now shown that the spikes, which, in the electron microscope, can be seen projecting from the surface of Rous virions, are removed by treatment with bromelain and their loss coincides with the loss of glucosamine containing proteins and of infectivity. Rifkin and Compans conclude therefore that the spikes of Rous sarcoma virions are glycoproteins necessary for infectivity and that they resemble the spikes of other enveloped viruses including flu and SV5 viruses.

\section{PROTEIN SYNTHESIS}

\section{Asserting the Fuith}

from our Molecular Biology Correspondent

From time to time a need arises within each field of research to provide the faithful with a sign. Some fifteen years since the word concerning the triplet code went forth, Gupta et al. (Biochemistry, 10, 4410; 1971) now offer more or less direct evidence that with each amino-acid incorporated into a polypeptide chain the ribosome does indeed, as everybody knew it would, slide, roll, bounce or lurch three nucleotide units along the messenger in the $5^{\prime}$ to $3^{\prime}$ direction.

Leaving out the separate processes of initiation and termination, the cycle of synthesis in bacterial systems is at present thought to go as follows: a quaternary complex is formed between a protein factor, GTP, an aminoacyltRNA and the A-binding site of the ribosome, sitting on its messenger, with the partly synthesized polypeptide chain, in the form of polypeptidyl-tRNA, at the other other (P) site. The GTP is then hydrolysed and the resulting GDP, still bound to the protein factor $\left(\mathrm{S}_{3}\right)$, as well as the orthophosphate that is formed, are liberated. The peptidyltRNA is hydrolysed, and the peptide chain makes a new peptide link with the amino-acid attached to its tRNA in the A-site. There is then a translocation step, evidently triggered by another ribosomal factor $\left(\mathrm{S}_{2}\right)$ with consumption of a further GTP, at the end of which the peptidyl-tRNA has returned to the $\mathrm{P}$ site, and the discharged tRNA has departed. Thereafter it is only necessary to regenerate a quaternary complex, a process which requires the intervention of a third factor $\left(S_{1}\right)$ to dissociate the $S_{3}-$ GDP, for the cycle to repeat. One takes it that GTP hydrolysis provides the mechanochemical urge to displace the ribosome along the messenger, but Gupta et al. point out that the implication of GTP at two points in the cycle leaves some doubt about the exact stage at which translocation occurs. They have accordingly examined the system frozen in turn at each state of the cycle.

The messenger is $\mathrm{f} 2$ phage RNA, and an initiation complex, involving ribosomes and formylmethionyl-tRNA, forms at the beginning of the coat protein cistron of the messenger. By adding or omitting the appropriate factors, $S_{1}$, $S_{2}$ and $S_{3}$, the system can be put into the pre- or post-translocation state. All of the messenger not in the ambit of the ribosome, and therefore protected by it, can then be trimmed away with nuclease. The remaining messenger can be dissociated from the ribosome, and its $3^{\prime}$ terminal sequence determined. The messenger trapped, after the digestion, in the initiation or the pre-translocation complex ends with the phenylalanine codon, UUU, corresponding to the fourth residue in the coat protein after formylmethionine. After translocation an additional triplet on the $3^{\prime}$ side is found to be protected. This is ACU,

\title{
Helium in Globular Cluster Stars
}

Globular clusters are of great importance because they are the oldest stellar systems in our galaxy. Thus, their ages may not only set a limit on the age of the galaxy, but may also provide an important clue to time scale over which the universe as a whole has evolved. A great deal of careful observation is necessary before such clusters can be accurately dated and determinations made of the distance to a cluster (and hence absolute luminosities), the composition of the stars in the cluster (particularly their helium and "metal" content) and the point at which the cluster members are leaving the main sequence. These data can then be related to theoretical evolutionary tracks for stars near the main sequence.

One bone of contention arising from such studies has been the exact choice of helium content necessary to fit the theoretical models to the observed properties of particular clusters. In next Monday's Nature Physical Science (January 10) John Faulkner, of the Lick Observatory, points out a convenient method for determining this from the exact shape of the evolutionary track followed by cluster members between the turn off from the main sequence and arrival at the giant branch.

Faulkner has looked at the best models now available for the cluster M 92. Between the main sequence and the giant branch, the rate of luminosity evolution of a star depends critically on its helium content, although in the main sequence and giant branch themselves composition is less important in this respect. This effect is largely independent of convection, the treatment of which is most uncertain in stellar evolution theory.

For M 92, Faulkner finds that comparison of the slope in the critical region with theoretical models of Simoda and Iben (Astrophys. J. Supp., 22, $81 ; 1970$ ) implies a helium content of $Y=0.33$. Values as low as $Y=0.2$ can be specifically excluded. This method of determining helium abundance by accurate measurement of just one portion of the evolutionary track of a cluster, rather than trying to relate all of the observations to a complete theoretical model, offers a powerful aid in the study of globular clusters. 